\title{
The Influence of Curly Grass Pattern in Chinese Traditional Architecture on Furniture Design
}

\author{
Lu Ting ${ }^{1, a}$, Dong Wenying ${ }^{1,2, b}$ \\ ${ }^{1}$ Sichuan agricultural University, College of Forest, Chengdu 611134, Sichuan, China \\ ${ }^{2}$ Central South University of Forestry and Technology, Changsha 410000, Hunan, China \\ a1291112993@qq.com, ${ }^{\text {b10775660@qq.com }}$
}

\begin{abstract}
Keywords: Furniture design, Traditional architecture, Curly grass pattern, Innovative
Abstract. With the quick pace of the development of cultural diversity, we pay more attention to the excavation of national characteristics. It is particularly urgent to dig deep to artistic feature and culture heritage of the traditional pattern with people aesthetic standards and the orientation of culture conception. Primary research the cultural background of the curly grass pattern and analyse the characteristics of the age of the curly grass pattern deduction with image collection and field visit etc. It summarizes the relationship between the the curly grass pattern and furniture design in traditional buildings which leads to the way of inheritance and innovation. And then gives some reference for develop and expand the national modern furniture products.
\end{abstract}

Countless design patterns are derived in different cultural background, dating back to ancient times. But as plant decorative pattern, the curly grass pattern play a big role in our history of art deco, and many other places of the world. It experienced the baptism and precipitate of the times from apply to Buddhist decorative art, and to building decorative pattern, and later to furniture design. Furniture become the main implements in the buildings, the two grow together, both serve for human creative activities of material.

\section{Cultural background of the curly grass pattern}

It has a long history of the curly grass pattern to be a Chinese ancient decorative pattern. It originated in ancient Greece, prevailed throughout medieval and flourished in Rococo and Renaissance. As Indian Buddhism spread to China in the Northern and Southern Dynasties, the curly grass pattern evolved by traditional acanthus design with originality. In Tang Dynasty, it is widely used in every field as important decorative pattern, such as building, lacquer-work, wall painting and brocade, made an enormous contribution to the flourish of Tang Dynasty's art. Actually, Chinese decoration art pay more attention to spiritual culture, not just simple symbolic and graphics. Decoration art focus on represent inner spirit and how to extract symbolic language, in this period. However, curly grass pattern satisfied people's aesthetic requirements of the form, spirit and meaning of decorative pattern with represent as a subject of plant.

\section{The curly grass pattern deducts characteristics of the age}

The curly grass patterns are always loved by the upper class of its diverse form, brilliant color, continuous vitality and smooth melodies. It contains the wisdom and emotion of nature, entrusts people's love of nature and pursuit of better life. Nowadays, it is also an emblem of simple, delicacy and freedom. It is integrated into new media visual art with different form, freely presented in various filed of art, such as indoor space, furniture design, clothing patterns and packaging advertisement.

The curly grass pattern is represents of the freely fashionable culture of the public. "Diversity of art means diversity of artistic aesthetic standard." Shouzhi Wang referred in "The World History of Graphic Design". Modern aesthetic idea becomes more romantic, unordered, free and tolerant under the influence of postmodern trend. The curly grass pattern become a visual sign of era culture and the 
symbolize of leading fashion active. It well loved with its alive and highlight form what changed people's emotion and polished their visually, and adapted to the need of times.

The curly grass pattern is a spiritual symbol of social upscale aesthetic culture.The curly grass patterns are always regarded as the symbol of elegant and wealthy life, whether period, or western and Chinese culture. Though its elegance fade, it still as the goal to pursuit delicacy alternative living of people due to its orderliness and the beauty of rhythm. It is widely used in architecture and furniture as delicate and classic element of decoration, satisfied the visual aesthetic and endowed cultural connotations of commodity at the same time. Nowadays, it is selected by a mass of high grade villa and houses. Rococo's curly grass pattern in the style of architectural to display the graceful humanistic life and settlement of high-grade building. Then it can say the curly grass pattern is a bridge linking both traditional and modern culture.

\section{The curly grass pattern decoration and furniture design in traditional structure}

Hegel once said "Only national, is the world". Furniture is an epitome of traditional structure, they are two bright pearls in the long history of China. Both were generated and developed in a long history and traditional culture of Chinese nation. They created structure and furniture culture with distinctive national identity, while satisfying human needs ${ }^{[1,2]}$.

The similar and different of the curly grass pattern in building and furniture

\section{On the adornment modelling and the component}

According to the difference of organization form and structure, the curly grass patterns could be divided into three types, such as independent structure, continuous structure and adaptive structure ${ }^{[3]}$. Independent structure could be divided into the free composition and corner structure with different position of decorate. In furniture's decoration, the free components of curly grass patterns usually be used on panel of independent decoration furniture and the back of a chair (Figure 1, the free components of the curly grass patterns are used on the back panel of a chair), but the corner structure were more used on decorate the corner with exclusive or combined use, such as the corner of furniture panel and the decoration of Spandrel (Figure 2, the corner structures of the curly grass patterns are used on furniture). We always use the corner structure on Sparrow Brace and Cover of architecture (Figure 3, the independent structures of the curly grass patterns are used on Sparrow Brace of architecture), but use the free composition on largish component like the Gate (Figure 4, the free components of the curly grass patterns are used on door panel, door carved plate), owing to flexible use of independent construction and adornment modelling of the curly grass patterns.

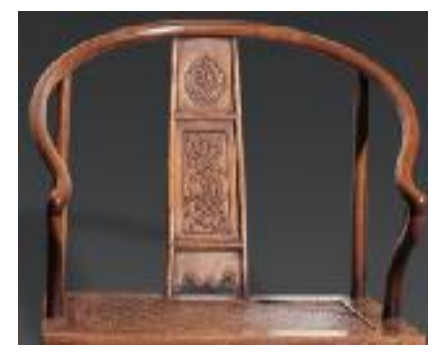

Fig 1. the free components of the curly grass patterns are used on the back panel of a chair

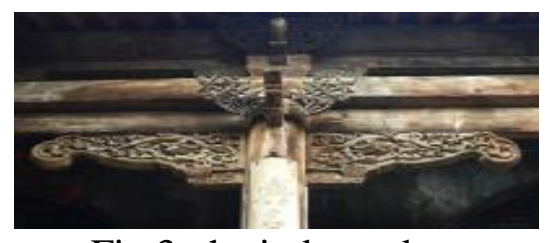

Fig 3. the independent structures of the curly grass patterns are used on Sparrow

Brace of architecture
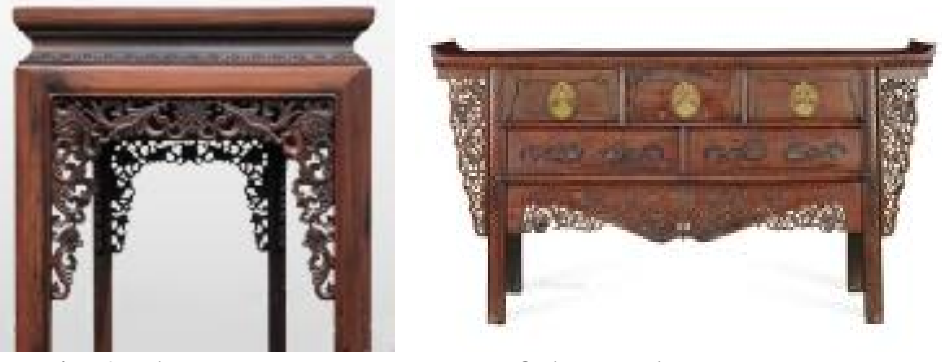

Fig 2. the corner structures of the curly grass patterns are used on furniture
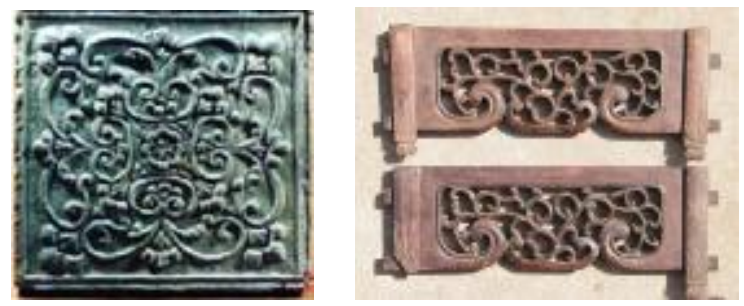

Fig 4. the free components of the curly grass patterns are used on door panel, door carved plate 
The most typical pattern of curly grass pattern decoration is continuous structure. It can be divide into two sides continual image and four consecutive units on the basis of different arrangement. Two sides continual image is the most common decoration of curly grass patterns in architecture and furniture, it is stretch and extend from left to the right. It is mainly applied to the structural component of banded architectures and furniture, such as Corbel Bracket, Capital Block, Flat Beam, Arhat Fang and Wooden Strip, etc (Figure 5, the curly grass patterns of two sides continual image are used on Sparrow Brace, Flat Beam, Cover). It is applied to decorate frame on furniture, such as Boor Shelter, Threaded Rods, Lattice and Bed Wai, etc (Figure 6, the curly grass patterns of two sides continual image are used on Bed Wai, Threaded Rods and the table legs, Spandrel and Three Bent Legs). Four consecutive units expanded though undulating left, right, up and down, it is harmonious and rhythmical. It is more used on fabric decoration than in architectural ornament.
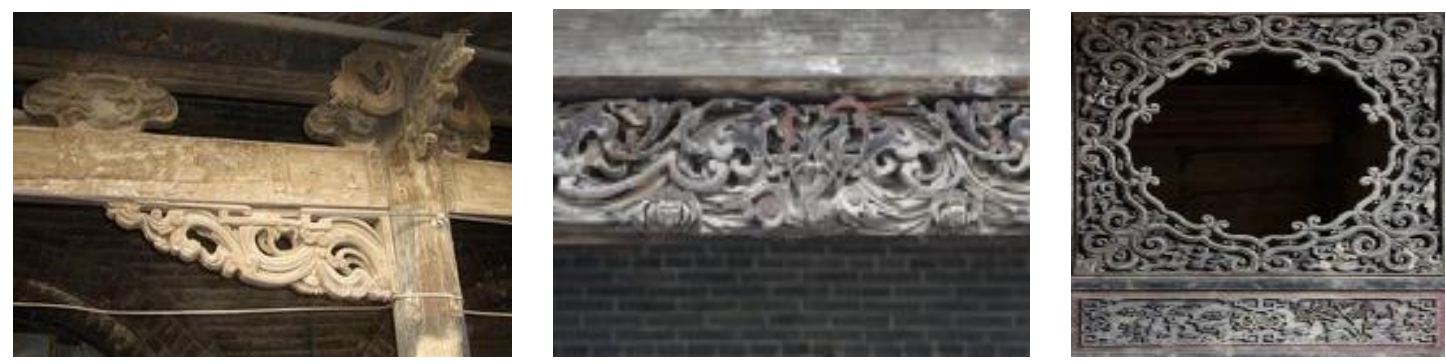

Fig 5. the curly grass patterns of two sides continual image are used on Sparrow Brace, Flat Beam, Cover
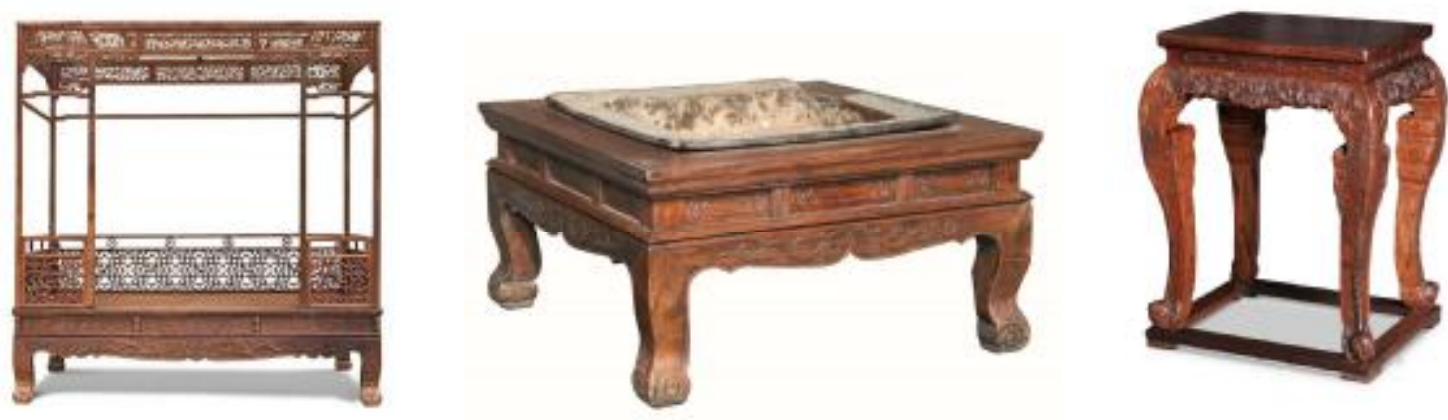

Fig 6. the curly grass patterns of two sides continual image are used on Bed Wai, Threaded Rods and the table legs, Spandrel and Three Bent Legs

of decorative space, having square, round and triangle in usual. It is rarely used on furniture due to its specific requirement of space, but is commonly used on niche decoration and in-out boxes of pottery of architecture.

The decorative element and expression

The curly grass patterns focused on various trans-formative fruits, flowers and leaves, regarded annular, waveform and interlace as the unit skeleton of the movement, freely extended to all around and perimeter. It made up a striking visual impact pattern which is independent and interrelated, refer to vines.

"Flowers are always regarded as the main pattern and cirrus serve for flowers in eastern curly grass patterns. The key point of pattern is the original symbolic meaning of flower that is connection by cirrus." Jocazo put forward in "The Contrast of Eastern and Western". As the main of the curly grass patterns, the importance of pattern's moral is easy to know. Such as: lotus of curly grass pattern represents auspicious and holy, is the symbol of "pure land", is widely used in architectural decoration of Buddhism; peony of curly grass pattern is a symbol of prosperity and happiness; prosperity grape leaves of curly grass pattern close to the family, is expected to offspring long desire; chrysanthemum flowers of the curly grass pattern mean long life and so on.

In traditional architecture, sculpture is almost everywhere you can see the decorative pattern of the curly grass patterns, which can be seen in different forms of wood carving in the structure of the folk 
house in southern Anhui province. On the brick walls of folk houses in the Jinzhong, you can see the lifelike brick carving. Which in the building of the wood carvings vividly in the furniture decoration in the performance--Line carving of the feminine, Relief carving of light, Openwork carving of ethereal and Round carving of the three-dimensional ${ }^{[4]}$. Take folk house in Huizhou as an example, in the lower parts of the window, the top of the Patio of the Fence, Eaves of the Floral scrolls decoration, there are more use Relief carving; However, on the decorative pattern of the curly grass patterns, such as Sparrow Brace, Corbel Bracket and Beam Drag, there are more use Round carving. The curly grass patterns are in the adornment of furniture parts, Relief carving is the most common woodcarving techniques, it to be used in large area to decorate. However, in the furniture on the smaller structural decoration, such as Bed Wai, Spandrel of Recessed-Leg Table, Sash Hanger Ring Plate and the through light components of Bottom Plates, etc, they are more use Openwork carving techniques. It's also used in part of the furniture, such as Throne, Washbasin stand with towel rack on both sides of the Top Rail, Hangers, carved the body or tail of Dragon and Phoenix with " $S$ " shape of the curly grass patterns, the capitals or squatting beast on top of Washbasin were in the shape of the curly grass patterns and Lotus pattern, as well as Clamp the Flower, etc, they are used in Round carving. The curly grass pattern is rarely used a Line carving alone in traditional building and furniture decoration, and it is commonly used with Relief carving (Figure 7, Relief carving, Openwork carving, Round carving are used on furniture parts).
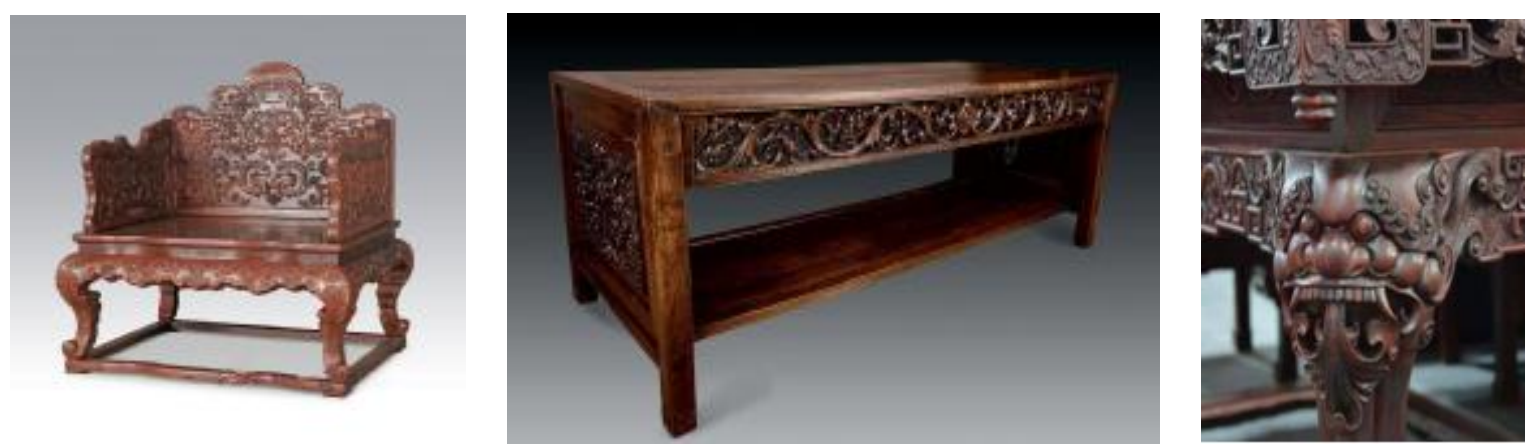

Fig 7. Relief carving, Openwork carving, Round carving are used on furniture parts

\section{The inheritance and innovation of the curly grass patterns in modern furniture}

It is easy to find that the curly grass pattern have a profound impact on the furniture decoration in traditional architecture, on the basis of analyse and summarize the different use of the curly grass patterns in traditional architecture and furniture in the view of the foregoing. However, it became a thinking matter for current designers that to expression curly grass patterns properly in modern furniture design. According to the research and thinking of the above, there are two ways to put forward:

Take its shape. Through the ages, there are ten thousand shapes and rich themes of the curly grass patterns. We should take out fussy element and make extract and abstract it on the basis of charm and essence of the curly grass patterns, pattern. The view have a strong visual impact and rhythm with exaggerate the flowers and leaves in proper through the resolve and reconstruct. Highlight the unique character and charm, meanwhile show a clear visual beauty of modern life.

Extending its meaning. The curly grass patterns already have the meaning of auspiciousness from "Liyue Zhi in Han Shu", and then honeysuckle pattern which present in Nan Bei Dynasty have the meaning of prolong life. The element of curly grass patterns express and inherit the good meaning with three common method to "symbolization, partial tone and table number" "5]. We are not only use the method in a good way in our design, also infuse new design element and the spirit of Meaning to extend deeper and more novelty spiritual idea and meaning, so that to convey the spirit of curly grass patterns and inheritance and development. 


\section{Conclusion}

The curly grass patterns become the eternal classic element of its natural, fluency and exquisite visual impact and highly applicable characters. The inseparable culture of traditional architecture and furniture not only represent the exquisite craft and remarkably intelligence of ancient labours, also represent the social and culture cognition of a nation and an ear. Only whole grasp and innovative inherit the traditional culture, can make the modern furniture organic integration with other culture, and provoke to initiate the new thinking for modern innovation and national art of contemporary designer.

\section{Acknowledgments}

This work was financially supported by the major project of humanities and social sciences of education department of Sichuan province (16SA0006), the "Key Laboratory of Wood Industry and Universities", the project of Undergraduate Scientific Research Interest of Sichuan Agricultural University.

\section{References}

[1] Fu Yue, The influence of traditional Chinese architecture on traditional Chinese furniture, Southwest Forestry University (2012.5).

[2] Qiu Zhitao, Ming Dynasty state-Research on the wisdom and value of Ming style furniture, Hunan People's Publishing House (2008).

[3] Wang Shuwen, Decorative features of curly grass patterns and its application in modern furniture design, Central South University of Forestry Technology (2013.6).

[4] Xu Yang, Ming and Qing furniture carving tools and process research, Northeast Forestry University (2007.4).

[5] Hu Zhongyan, Cao Yang, The inheritance and development of ancient Chinese furniture design, Packaging Engineering, Vol. 30,01 (2009), p.158-159. 